\title{
LA PUBLICIDAD EN LA PROGRAMACIÓN DEPORTIVA DE LA RADIO ESPAÑOLA: UN ANÁLISIS CON ENFOQUE DE GÉNERO ${ }^{1}$
}

\author{
Eva Gil ${ }^{2}$
}

\begin{abstract}
La publicidad en la programación deportiva de la radio española: un análisis con enfoque de género

Resumen: Las características de la programación deportiva en la radio española hacen de ella un ámbito realmente interesante para la investigación sobre la publicidad radiofónica, sobre todo desde la perspectiva de género. En este artículo constatamos la representación publicitaria de las mujeres en esta programación deportiva, espacio ciertamente masculinizado, conclusión a la que llegamos después de detallar diferentes aspectos de su publicidad, como las voces o el lenguaje utilizado. Para ello, nos basamos en los anuncios incluidos en los distintos programas de deporte de emisoras variadas, los cuales han sido recopilados durante los años 2004, 2006/07 y $2009 / 10$.
\end{abstract}

Palabras Clave: publicidad, radio, género, deportes, voces, sexismo, androcentrismo, lenguaje.

Advertising on Sports Programming in Spanish Radio: An Analysis from a Gender Perspective

Abstract: The characteristics of sports programming in Spanish radio make a really interesting field for research on radio advertising, especially from the perspective of gender. In this article, we can see the advertising representation of women in sports programming, certainly a masculinized sphere. We reached this conclusion after analysing different aspects of advertising, as the voices or the language used. For this purpose, we base our research on ads included in different sport programs from varied stations, which have been collected during the years 2004, 2006/07 and 2009/10.

Key words: advertising, radio, genre, sport, voices, sexism, androcentrism, language.

\section{Introducción}

Las especiales características de la programación deportiva en la radio española la convierten en un ámbito muy interesante para la investigación sobre la publicidad

\footnotetext{
${ }^{1}$ Fecha de recepción: 23/08/2014.

Fecha de aceptación: 14/09/2014.

El presente texto sobre la publicidad radiofónica en la programación deportiva española forma parte de la tesis doctoral "Mujeres y hombres en el discurso publicitario radiofónico (2004-2010)", defendida por la autora el día 24 de octubre de 2013 en la Universidad de Málaga y que obtuvo una calificación de sobresaliente cum laude.

${ }^{2}$ Tesorera de la Asociación de Estudios Históricos sobre la Mujer, Universidad de Málaga, España; $\square$ aehm@uma.es.
} 
radiofónica, sobre todo desde la perspectiva de género (Gil 2012a: 133-148). Por ello, en el siguiente estudio se analizarán los anuncios incluidos en los distintos programas de deporte de las emisoras elegidas a lo largo de tres recopilaciones, en los años 2004, 2006/07 y 2009/10: se trata de retransmisiones deportivas especiales, magacines de fin de semana o programas de noticias en la Cadena Ser, Cadena Cope, Onda Cero, Punto Radio, Radio Nacional de España y Canal Sur Radio; en el caso de los años 2009/10 contamos, además, con la programación de una emisora dedicada al deporte, Radio Marca, y otra de carácter "mixto", Rock\&gol, en la que destacan fundamentalmente los deportes y la música.

El objetivo fundamental de este artículo es constatar la representación de las mujeres en la publicidad emitida durante la programación deportiva radiofónica española. Para ello, se ha estructurado en cuatro apartados fundamentales, como son la voz en los anuncios, los productos publicitados, la presencia de uno u otro sexo en esta publicidad y el lenguaje utilizado en esta. Se trata de aspectos que nos terminan demostrando que estamos en un ámbito centrado de forma mayoritaria en la figura masculina. Metodológicamente, se incidirá en el análisis de los datos obtenidos en la recopilación de anuncios de 2009/10, que resulta la más completa y minuciosa de las tres, y se utilizarán las de 2004 y 2006/07 como punto de comparación, con la idea de observar una posible evolución en los resultados de cada uno de los mencionados epígrafes.

\section{Revisión teórica: mujer y deporte/mujer y publicidad radiofónica}

Desde que en 2004 comencé a investigar sobre publicidad radiofónica, uno de los aspectos que más me ha llamado la atención ha sido la singularidad de la publicidad emitida durante la programación deportiva, un "mundo aparte" sin lugar a dudas, lo que siempre me llevó a considerarla casi como una franja horaria más, diferenciada de las habituales. José Luis Rojas Torrijos (2010: 6) apunta que dentro del ámbito informativo, 
la comunicación deportiva está considerada como una de las manifestaciones periodísticas que de forma más clara transmite estereotipos sexistas.

Ana Ma Vigara (2008: 113-160), Ma Elena Gómez (2010: 115-132) o José Luis Rojas (2010: 1-15) denuncian la invisibilidad del deporte femenino, que no se muestra ni se apoya en los medios de comunicación. En el corpus documental que le sirve de base a su artículo, Rojas (2010) apenas encuentra un 1\% de noticias que recogieran resultados de competiciones deportivas femeninas: ello conlleva, además una escasa presencia de mujeres en la comunicación deportiva, permitiendo todavía desigualdades, como las del lenguaje que se emplea en este ámbito. Vigara (2008: 116-125), sin embargo, considera que "más que sexismo propiamente lingüístico, lo propio del periodismo deportivo es sexismo informativo". En este sentido, además, como plantea Gómez Sánchez (2010: 118), no podemos obviar en el análisis del discurso mediático sobre las mujeres en el periodismo deportivo la mirada de quienes, tradicionalmente, han sido y son responsables de este tipo de información y también la de quienes, "tradicional y mayoritariamente", la han recibido: los hombres.

Los trabajos anteriormente citados de Vigara (2008), Gómez (2010) y Rojas (2010) se centran en la prensa, pero no podemos olvidar que el deportivo es un género radiofónico que también está en alza $^{3}$, de ahí que la facturación publicitaria en estos espacios sea de las más altas de la radio en España (Herrero y Romero 2011: 235-236). Son circunstancias que, desde un primer momento, me han hecho considerar que la publicidad emitida durante la programación deportiva merece su estudio propio.

Lo cierto es que, en general, no parece que la publicidad radiofónica sea especialmente sexista; o por lo menos así podemos deducirlo de las escasas denuncias que recibe este medio en organismos como el Observatorio de la Imagen de las Mujeres del Instituto de la Mujer o el Observatorio Andaluz de Publicidad no Sexista. Pero el sexismo de la publicidad radiofónica, de forma general y muy especialmente en su

\footnotetext{
${ }^{3}$ Francisco Javier Herrero Gutiérrez y David Rodríguez Ramos (2009: 970), citando a José María Cagigal (Deporte: espectáculo y acción, Barcelona, 1981), consideran que las parrillas de las grandes emisoras radiofónicas conceden un espacio cada vez mayor a las noticias deportivas: "si se conecta la radio un día cualquiera, pronto podrá oírse un noticiario deportivo, y si aquella se sintoniza en domingo, puede decirse sin exageración que será difícil escuchar otra cosa".
} 
programación deportiva, no viene marcado por una tendencia a utilizar palabras que puedan resultar insultantes para sus oyentes: la sociedad ya no se lo permitiría. También, como acertadamente expresa Ma Ángeles Pérez Chico (2002: 43): "no parece muy lógico que un anunciante busque de forma gratuita la ofensa de sus potenciales compradores". Sin embargo, se puede valer de otras estrategias para ocultar la presencia de las mujeres y reducir su valor como posibles interesadas en los productos que oferta, convirtiendo a la figura masculina en el centro indiscutible de la publicidad emitida en los programas y las retransmisiones deportivas. En este sentido, se puede hablar de androcentrismo, más que de sexismo propiamente dicho.

\section{La voz en la publicidad radiofónica deportiva}

La primera pregunta que nos podemos plantear es: ¿de quién es la voz en la publicidad que se emite en la programación deportiva?

La recogida de datos que se llevó a cabo a lo largo de 2009-2010 no arroja cifras esperanzadoras. A lo largo de 62 horas y 10 minutos se grabaron los siguientes programas: "El larguero", "Carrusel deportivo" y "Ser deportivos", de la Cadena Ser; "Radio Estadio" y "Al primer toque", de Onda Cero; "El tirachinas", de la Cadena COPE y "El mirador de la liga", de Punto Radio. En este espacio temporal también encontramos una programación deportiva especial, aprovechando la participación de la selección nacional de fútbol en un campeonato internacional, con dos retransmisiones dedicadas exclusivamente a estos partidos: una de ellas en la Cadena Ser y la otra en Onda Cero.

En esta recopilación de anuncios resultan interesantes dos emisoras con un carácter eminentemente deportivo: Rock\&gol y Radio Marca. Vinculadas a cadenas generalistas (Cadena COPE y Onda Cero), todas sus emisiones no están dedicadas solo al deporte, ya que pueden incluir programas de música o contenidos relacionados con la televisión o el cine. Aun así, en ambos casos he considerado conveniente la inclusión en este apartado dedicado al deporte de toda la programación grabada: las dos emisoras se convierten en 
el lugar para las retransmisiones deportivas locales de estas cadenas, repeticiones o emisiones simultáneas de espacios de deportes, además de sus propios programas, dedicados sobre todo al mundo del fútbol o del motor. Que haya un cierto respiro con la música y demás ocio parece también necesario para dotar a su programación de cierta variedad, sin perder con ello a lo que se considera su público objetivo: el masculino.

La voz en la publicidad de radio que se emite en estos programas es, en más de una tercera parte, masculina $(76,1 \%)$, en tanto que la voz femenina apenas supera el $5 \%$ $(5,1 \%)$. Cuando ambas voces comparten un mismo espacio publicitario, la voz masculina comienza el diálogo en el 11,3\% de los casos y la femenina, en un 6,2\%. El porcentaje restante queda repartido en las ocasionales intervenciones de voces infantiles junto a la de adultos, también masculinas en su mayoría. De esta manera, las cifras totales son demoledoras: en casi el 95\% de los anuncios grabados durante la programación deportiva aparece al menos una voz masculina, mientras que solo sucede lo mismo con la femenina en apenas el $23 \%$ de los casos.

La tabla 1 ofrece unos números muy interesantes, que nos permiten comparar la presencia de ambos sexos en la publicidad de la programación deportiva frente a los valores calculados para el total de la publicidad que compone la muestra del año 2009/10 en el resto de las franjas horarias y temáticas ${ }^{4}$.

TABLA 1. RECOPILACIÓN 2009/10

\begin{tabular}{|l|l|l|}
\hline VOCES & $\begin{array}{l}\text { PORCENTAJES } \\
\text { TOTALES }\end{array}$ & $\begin{array}{l}\text { PROGRAMACIÓN } \\
\text { DEPORTIVA }\end{array}$ \\
\hline VOZ MASCULINA & $59,20 \%$ & $\mathbf{7 6 , 1 0 \%}$ \\
\hline VOZ FEMENINA & $10,00 \%$ & $\mathbf{5 , 1 0 \%}$ \\
\hline
\end{tabular}

\footnotetext{
${ }^{4}$ Datos procedentes de la tesis doctoral de la autora, "Mujeres y hombres en el discurso publicitario radiofónico (2004-2010)", pendiente de publicación.
} 


\begin{tabular}{|c|c|c|}
\hline $\begin{array}{l}\text { VOCES } \\
\text { FEMENINA }^{5}\end{array}$ & $15,00 \%$ & $11,30 \%$ \\
\hline $\begin{array}{ll}\text { VOCES } & \text { FEMENINA/ } \\
\text { MASCULINA }^{6} & \end{array}$ & $14,50 \%$ & $6,20 \%$ \\
\hline OTROS & $1,30 \%$ & $1,30 \%$ \\
\hline
\end{tabular}

En mi opinión, hay dos motivos que pueden ayudar a explicar esta escasa presencia de mujeres en la publicidad de la radio deportiva española. En primer lugar, la publicidad parece resistirse a una idea que resulta evidente: a las mujeres también les gusta el deporte, quieren mantenerse informadas y divertirse con este tipo de programación. No se han encontrado cifras de audiencias desagregadas por sexo pero no hace falta más que poner la radio y prestar atención a los mensajes vía telefonía móvil o Internet que manda la gente para hacer comentarios: nos daremos cuenta de que también las mujeres participan a través de estos medios.

En segundo lugar, apenas hay voces femeninas en esta publicidad porque tampoco las hay en los equipos que realizan los programas deportivos. En la publicidad de algunos de estos espacios podemos distinguir entre las tradicionales cuñas enlatadas $\mathrm{y}$, sobre todo, la publicidad que realizan en directo a lo largo de todo el programa, con "animadores" como Pepe Domingo Castaño o comentaristas como Kiko Narváez ${ }^{7}$. En ella, además, intervienen otros compañeros del equipo, por lo que aumentan notablemente el número de voces masculinas. Las mujeres, que pueden ser periodistas en labores de ayuda en la retransmisión de los partidos o público asistente a la emisión del programa, cuando aparecen en este tipo de anuncios, se limitan a dar un toque sensual.

\footnotetext{
${ }^{5}$ Anuncios en los que encontramos voces de ambos sexos, aunque es la masculina la que comienza el diálogo.

${ }^{6}$ Anuncios en los que encontramos voces de ambos sexos, aunque en este caso es la femenina la que comienza el diálogo.

${ }^{7}$ Sobre la presencia y la importancia que está adquiriendo la figura del "animador" en la publicidad de la programación deportiva (sobre todo cuando se trata de retransmisiones en directo), véase el artículo de Francisco Javier Herrero Gutiérrez e Hilario José Romero Bejarano (2011).
} 
En la recopilación realizada a finales de 2006 y principios 2007, los programas grabados fueron "Carrusel deportivo", de la Cadena Ser; el espacio deportivo incluido en el programa "La brújula" de Onda Cero; "El Tirachinas", de la Cadena COPE; "El pelotazo", de Canal Sur Radio y "Radio gaceta de los deportes", de Radio Nacional de España. La tendencia es muy clara: el $84,3 \%$ de los anuncios emitidos en estos espacios están protagonizados por voces masculinas y el $0 \%$ por voces femeninas. En el 12,75\% de los casos, voces masculinas y femeninas comparten campaña publicitaria, mientras que en un 1\% sucede al revés, con la mujer iniciando el diálogo. En el casi 2\% restante, aparecen voces masculinas y femeninas junto con niños o niñas.

En la toma de datos que se realizó en el año 2004, tres emisoras fueron objeto de estudio: Cadena Ser, Onda Cero y Cadena COPE. Los programas deportivos grabados en estas cadenas fueron "Carrusel deportivo", "Radio estadio" y "Tiempo de juego", respectivamente. En este caso, las cifras fueron también esclarecedoras: la voz que se escucha en el $72,5 \%$ de los anuncios emitidos es exclusivamente masculina, mientras que solo hay un 5,625\% en los que es femenina. Cuando comparten espacio más voces, en el $13,75 \%$ de los casos empieza el diálogo una voz masculina y le responde una voz femenina y en casi el 7\% sucede lo contrario: es la voz femenina la que comienza a hablar. Hay también una presencia testimonial de anuncios en los que las voces son de niño-padre $(1,25 \%)$, pero nunca de niña-padre o niño/a-madre. Es decir, que si sumamos los anuncios en los que aparece alguna voz femenina, nos encontramos que lo hacen en algo más de la cuarta parte de la publicidad emitida durante la programación deportiva $(26,25 \%)$. La voz masculina, en este caso, es incuestionable, ya que se encuentra en el 94,375\% de los anuncios grabados.

Así, cinco años después (2004-2009), la situación, lejos de mejorar gracias a una mayor concienciación ciudadana, leyes o recomendaciones de los organismos públicos, se mantiene casi en los mismos niveles que encontré en el año 2004. Los porcentajes ponen de manifiesto la escasa consideración de la "variable femenina" en la dirección de la publicidad de un tipo de programación como esta, que se centra en la retransmisión y los comentarios de fútbol, y que parece diseñada con un claro carácter masculino (Arenas et al. 2003: 202). 


\section{Los productos objeto de la publicidad deportiva}

Por lo que respecta a los productos objeto de esta publicidad, se puede decir que todos los años encontramos un panorama muy fragmentado, señal del interés que genera este espacio publicitario para posibles anunciantes.

En la recopilación de 2009/10 se recogen hasta cuarenta y ocho categorías diferentes que, en muchos casos, engloban más de un producto, aunque siempre de similares características.

La publicidad más repetida en los espacios deportivos grabados es la relacionada con los coches. Se trata de un dato que también confirma Álvarez Villa (2004: 177), que denomina a la publicidad sobre automóviles "el producto rey en los bloques publicitarios que aparecen en el género magacín deportivo". Le sigue en importancia la autopromoción que realizan las emisoras de sus características o de los contenidos de sus diferentes programas, tanto de los deportivos como los de otras franjas horarias.

En tercer lugar, nos encontramos con la restauración y demás locales relacionados con el ocio. Y ya, a mayor distancia, aparece un anunciante, las agencias de viaje, cuya posición está muy condicionada por las fechas en las que ha tenido lugar la toma de datos: entre abril y agosto, fundamentalmente, lo cual significa que incluye tanto las vacaciones de Semana Santa como las de verano.

En los primeros puestos de la tabla tenemos también las tiendas y los centros comerciales. Cuando hablamos de tiendas, destacan sobre todo aquellas que podríamos considerar dedicadas a la renovación "a gran escala" del hogar: puertas, muebles, azulejos, cristalería, etc. Por lo que respecta a los centros comerciales, se intenta captar la atención masculina:

[Voz femenina]: ¿Quieres estar fantástico?

[Voz masculina]: Ven a la Semana Fantástica y llévate lo mejor del verano. 
[Voz femenina]: Dos camisas de manga corta o dos bermudas de Emidio

Tucci, por solo [...].

[Voz masculina]: Y en primeras marcas de moda, descuentos de hasta el 30\%.

[Voz femenina]: Semana Fantástica en El Corte Inglés ${ }^{8}$.

Seguros, empresas financieras y bancos forman también una terna muy repetida en la publicidad de la programación deportiva. Con el término "empresas financieras" quería hacer referencia a una serie de negocios que se dedican a agrupar préstamos, dar créditos o ayudar al pago de hipotecas y que han proliferado de manera especial en la actual recopilación.

Como podemos comprobar, si dejamos a un lado la publicidad de autopromoción radiofónica, los conceptos más repetidos están relacionados todos ellos con aspectos que tradicionalmente se han reservado a la actuación de la figura masculina: el coche y su mantenimiento, el ocio, las adquisiciones que implican un mayor desembolso de dinero para el hogar y decisiones económicas de importancia.

En la recopilación anterior a la comentada, realizada entre finales de 2006 y principios de 2007, la publicidad emitida durante el horario dedicado a los deportes presentaba los siguientes productos: vehículos comerciales, carrera popular, restauración, parquet, grandes almacenes, centros comerciales, tarjetas de UNICEF, coches y sus complementos, coches todoterreno, taller de coches, tienda de puertas y armarios, de muebles, de charcutería, de tecnología, de menaje de hogar, casas móviles, ONCE, bebidas alcohólicas, talón-hotel, motocicleta (promoción de un diario deportivo), tarjetas de crédito, información telefónica, centro de vacaciones, zumos, pipas, puesto informático, Correos, tractores, teléfonos móviles, cemento, televisión digital, espectáculo de magia, espectáculo de danza, videojuegos, programación de la radio, ciclo de conferencias sobre la mujer, Renfe, retransmisiones deportivas, encuesta, Internet,

\footnotetext{
${ }^{8}$ En “Retransmisión de fútbol”, Onda Cero, 28 de junio de 2009. Horario: de 14: 50 a 16:00 h.
} 
cursos de formación para profesionales del mundo rural y otros intangibles, como la información sobre el cierre de Air Madrid, las campañas de la Dirección General de Tráfico y la Fundación de Ayuda contra la Drogadicción. Aunque la variedad de productos es interesante y tampoco se puede afirmar rotundamente que unos productos se orientan en exclusividad a los hombres y otros a las mujeres, lo cierto es que más que en ninguna otra franja horaria, se incide en la compra de vehículos comerciales, utilitarios de gama alta, en los talleres para coches y en productos tan especializados como el cemento o, incluso, los tractores.

De la misma manera, en el análisis de la publicidad recopilada en el año 2004 obtenemos una idéntica conclusión: la publicidad en la franja horaria deportiva es eminentemente masculina; está protagonizada por hombres, se dirige a ellos y les ofrece aquellos productos que más les interesan: los "puritos" (todavía estaba permitida la publicidad de tabaco), prensa deportiva, revistas masculinas, bebidas alcohólicas, coches, telefonía móvil, sorteos, tarjetas bancarias...

Al ser predominante la voz masculina, la podemos encontrar en la publicidad de prácticamente todos los productos. En cambio, la voz femenina solo se dedica en exclusiva a los siguientes conceptos:

1. La promoción turística9:

[Voz femenina]: Una excusa para juntaros: hoy monte y mañana playa. Y al otro, un pueblecito de paredes blancas, unas olas para cabalgar, un baño de burbujas y un brindis porque esto se repita. Un viaje memorable en Castellón Costa Azahar. Comunidad Valenciana. Te doy todo. Generalitat.

2. La promoción cultural ${ }^{10:}$

[Voz femenina]: Con Extremúsica cada año llega la primavera. Lo verás del 16 al 18 de abril en Mérida. ¿Un adelanto a nuestra bonita locura? Ya sabes [...], Rosendo, The Toy Tolls. Hay más: Mago de Oz, Ilegales [...]. En fin,

\footnotetext{
9 En "Radio Estadio", Onda Cero, 5 de abril de 2009. Horario: de 16:00 a 18:00 h.

10 En Rock\&gol, 3 de abril de 2009. Horario: de 15:00 a 20:00 h.
} 
más de setenta bandas, homenaje al rock andaluz y concentración motera. En Extrarruta. Tus entradas en Tic Tac tickets. Extremadura en abril. Eso es música. A la vera del Guadiana y de Rock\&gol.

\section{Los cuidados médicos ${ }^{11:}$}

[Voz femenina]: Si está pensando en colocarse un implante dental, no lo haga sin consultar antes con la clínica dental Avandent. Porque en Avandent subvencionamos parte de su implante dental a fondo perdido. Sí, ha oído bien: a fondo perdido. Infórmese ya [...].

\section{El ocio ${ }^{12}$}

[Voz femenina]: Bar Aguanta. Desde 1989 apoyando al Málaga en La Rosaleda. Ven a Bar Aguanta. Por las tardes, juega a las cartas o a los dardos. Por la noche, ven a tomar una copa y a disfrutar de la música [...].

\section{La restauración ${ }^{13:}$}

[Voz femenina]: Bodegón Pajarete. Nuestra especialidad es la cocina típica malagueña en tapas y raciones regadas con los mejores vinos andaluces [...].

6. Tiendas y centros comerciales:

-De colchones ${ }^{14}$ :

[Voz femenina]: Ahora sí, en Mi colchón puedes ver unos precios que no verás jamás: canapé de madera y colchón viscoelástico de matrimonio por solo [...] O vienes o te lo pierdes.

- De pinturas ${ }^{15}$ :

11 En "El larguero", Cadena Ser, 4 de abril de 2009. Horario: de 24:00 a 1:30 h.

${ }^{12}$ En "Carrusel deportivo" (especial con la retransmisión del partido España-Estados Unidos), Cadena Ser, 24 de junio de 2009. Horario: de 20:00 a 22: $30 \mathrm{~h}$.

${ }^{13}$ En "Directo Marca", Radio Marca, 17 de agosto de 2009. Horario: de 9:00 a 12:00 h.

${ }^{14}$ En "Carrusel deportivo", Cadena Ser, 18 de abril de 2009. Horario: de 18:00 a 23:00 h

${ }^{15}$ En "El tirachinas", Cadena COPE, 28 de abril de 2009. Horario: de 24:00 a 1: 30 h. 
[Voz femenina]: Pinturas Málaga Sol ha inaugurado un nuevo punto de venta en el polígono comercial [...] Unas instalaciones más cómodas, más amplias, con más de 500 metros cuadrados de exposición al público. Todo primeras marcas en el mundo del color [...] Pinturas Málaga Sol. Pinta con nosotros. Pinturas Málaga Sol. A todos, felicidades.

\section{Peluquería ${ }^{16:}$}

[Voz femenina]: Agustín e hijos peluqueros. Centro comercial Larios, Rosaleda y en el centro histórico.

\section{Productos médicos y de parafarmacia ${ }^{17}$}

[Voz femenina 1]: Estoy agotada de estudiar. Mi memoria ya no da para más.

[Voz femenina 2]: Recupera tu memoria con Dememory. Te tomas una ampolla diaria de Dememory y memoria de elefante. Recuerda, refuerza tu memoria con Dememory. En tu farmacia.

9. Telefonía e Internet ${ }^{18:}$

[Voz femenina]: Con respuesta profesional tienes un mini PC para conectarte donde quieras, con banda ancha, móvil, desde 39,90 euros al mes los seis primeros meses [...] Respuesta Profesional de Telefónica.

\section{Campañas de tráfico ${ }^{19:}$}

[Voz femenina]: A los sistemas habituales para el pago de multas de tráfico ahora se suma Internet [...] Internet al servicio de los conductores. Dirección General de Tráfico [...].

\footnotetext{
${ }^{16}$ En "Carrusel deportivo", Cadena Ser, 18 de abril de 2009. Horario: de 18:00 a 23:00 h.

${ }^{17}$ En "El café del paddock", Radio Marca, 30 de abril de 2009. Horario: de 16:00 a 20:00 h.

${ }^{18}$ En "El larguero", Cadena Ser, 15 de mayo de 2009. Horario: de 24:00 a 1:30 h.

${ }^{19}$ En "Retransmisión de fútbol", Onda Cero, 28 de junio de 2009. Horario: de 14: 50 a 16:00 h.
} 
11. Asociaciones ${ }^{20}$

[Voz femenina]: Las familias impulsan el movimiento asociativo FEAC.

12. La automoción ${ }^{21:}$

[Voz femenina]: IMSA, su concesionario oficial AUDI en Málaga [...].

13. La autopromoción de la programación radiofónica 22 :

[Voz femenina]: Rock\&gol. Lo mejor de la música y el deporte.

Se trata de apenas trece conceptos, frente a los cuarenta y ocho que componen el total de dicha muestra (2009/10): es todavía un porcentaje demasiado escaso para considerar que estamos ante una tendencia evolutiva favorable.

\section{5. ¿Androcentrismo publicitario?: Mujeres y hombres en la "publicidad deportiva"}

Ya hemos visto a través de las cifras el carácter eminentemente masculino de la publicidad que se emite en la programación deportiva de la radio española, pero con los siguientes ejemplos podemos incidir en otros aspectos también muy interesantes:

a.El deporte se convierte en el espacio ideal para que el hombre se fije en su apariencia y en su bienestar físico:

- Para la mujer, el uso de cosméticos se convierte en una necesidad para luchar contra el paso de los años; para el hombre no es un problema de edad, sino de cansancio. O por lo menos así parece deducirse de los siguientes anuncios, perfecta contraposición y ejemplo de la forma diferente de dirigirse a mujeres y hombres en un caso muy específico como es el de los productos cosméticos:

\footnotetext{
${ }^{20}$ En "El larguero", Cadena Ser, 6 de agosto de 2009. Horario: de 24:00 a 1: $30 \mathrm{~h}$.

${ }^{21}$ En "Directo Marca", Radio Marca, 17 de agosto de 2009. Horario: de 9:00 a 12:00 h.

${ }^{22}$ En "Música", Rock\&gol, 31 de enero de 2010. Horario: de 23:00 a 23:30 h.
} 
[Voz masculina]: Cuando estás cansado, tu piel se apaga, pierdes energía, te vuelves gris. Recárgate con High Natural Brown, el nuevo hidratante antifatiga de Biotech que día tras día da a tu piel un buen tono de forma natural. El secreto de tu buen color. Biotech. Porque un buen aspecto contribuye al éxito ${ }^{23}$.

[Voz femenina]: Hola, soy Laura. ¿Qué por qué uso Elastovital? Porque no tengo 20 años y mi piel necesita algo más que la típica crema cosmética: por eso utilizo la crema Elastovital y además me tomo las cápsulas también de Elastovital y, chica, yo no sé si es la crema o las cápsulas pero con Elastovital recuperas esa juventud que se te escapa con la edad ${ }^{24}$.

- Mientras que en franjas horarias como la matinal o la de tarde, la preocupación se centra en "arreglar" los problemas que acucian a las mujeres: con su peso, con su falta de energía o con la llegada de la menopausia; en la deportiva, estos consejos desaparecen y se convierte en el espacio en el que los hombres pueden buscar la solución a problemas como la pérdida del cabello, los ronquidos o dificultades sexuales. En los siguientes anuncios, además, encontramos una circunstancia muy llamativa: son ellos los afectados, aunque las mujeres tienen que soportarlo de la misma manera y, en algunos casos, incluso tomar la iniciativa para su solución:

[Voz femenina]: Desde luego, ya no eres el de antes. ¿Has visto la cantidad de pelo que estás perdiendo?

[Voz masculina]: Pues claro que lo veo, todo el mundo me lo dice. ¿Qué voy a hacer? Esto no hay quien lo pare.

\footnotetext{
23 Ibidem.

${ }^{24}$ En "Hoy es domingo", de Onda Cero, 8 de febrero/7 de marzo de 2004. Horario: de 9:00 a 12: 30 h.
} 
[Voz femenina]: Sí hombre, lo que tú digas. Eso sería antes. Yo me he molestado en pedir consejo en la farmacia y me han hablado de Keren 2, un producto que mantiene y regenera el cabello.

[Voz masculina]: No sé, cariño, ¿tú crees que me valdrá?

[Voz femenina]: Ya lo creo. Venga, vamos a la farmacia que te lo expliquen. $[\ldots]^{25}$. $[\ldots]$

[Voz masculina 2]: Oye, ¿te ha cambiado la vida el Snord Stopper?

[Voz masculina 3]: Pues sí, la verdad es que sí. Ahora ya por las noches no se ronca y la verdad es que a quien había que preguntárselo es a mi mujer, porque ya puede dormir en la cama y sin tapones. Cansado ya de los codazos de la mujer [...] lo oí en Radio Marca y decidí probarlo [...].

$* * * * * * * * * * * * * * * * * * * *$

$[\ldots]$

[Voz masculina 3]: [...] Lo he recomendado a varios familiares, amigos que también roncan y, vamos, he hablado con las mujeres y enseguida se van a poner en contacto para adquirir ya este producto $[\ldots]^{26}$.

b. Para comprobar que la publicidad durante la programación deportiva de la radio española está pensada, preferentemente, para un público objetivo, el masculino, podemos tomar como referencia un producto que cuenta con una amplia difusión en

\footnotetext{
${ }^{25}$ En "El tirachinas", Cadena COPE, 28 de abril de 2009. Horario: de 24:00 a 1: $30 \mathrm{~h}$.

${ }^{26}$ Los dos últimos anuncios en "Directo Marca", Radio Marca, 17 de agosto de 2009. Horario: de 9:00 a $12: 00 \mathrm{~h}$
} 
diferentes franjas horarias y programas: Revital, un "complemento energético que ayuda a mantener bienestar, energía y vitalidad"27. Pues bien, estas campañas suelen estar protagonizadas por voces masculinas y femeninas, unas veces famosos como Jimmy Giménez Arnau o Concha Cuetos, aunque la mayoría representan a personas anónimas que cuentan el por qué de su necesidad y los beneficios que les reporta la ingesta de este producto. Así, en el programa de "La mañana", de la Cadena COPE, una voz femenina es la que nos proporciona su experiencia:

[Voz femenina]: ¿Que por qué tomo Revital? Pues porque me mantiene despierta, muy en forma. Por las mañanas me tomo mi ampolla de Revital y me da energía para todo el día, trabajar, entrar, salir, la casa, los niños. Uy, parezco superwoman. No sé, Revital me da mucha energía ${ }^{28}$.

En otra cuña, esta vez en horario de noche, el argumento femenino del trabajo y el cuidado familiar se repite:

[Voz femenina]: ¿Qué por qué tomo Revital? Pues yo tomo Revital sobre todo cuando estoy cansada. Mira, entre el trabajo, el marido, los niños, la compra. Es que acabo agotada. Pero es que tomo una ampolla de Revital y me quedo nueva, con más fuerza, con más energía. No sé, pero con Revital es que me siento otra ${ }^{29}$.

En los espacios deportivos ya no hay mujeres que cuenten una atareada vida familiar y laboral: los protagonistas siempre van a ser hombres y las necesidades están más bien relacionadas con el ocio y el placer $^{30}$ :

\footnotetext{
27 Información obtenida en la página web del producto: http://www.pharmaotc.com/f2_Revital.html.

${ }^{28}$ En "La mañana de la COPE", Cadena COPE, 28 de abril de 2009. Horario: de 5:00 a 8:00 h.

${ }^{29}$ En "La brújula", Onda Cero, 11 de mayo de 2009. Horario: de 20:00 a 24:00 h.

${ }^{30}$ Los dos anuncios en "El café del paddock", Radio Marca, 30 de abril de 2009. Horario: de 16:00 a 20:00 h.
} 
[Voz masculina]: ¿Crisis energética? Con Revital, ¿desde cuándo? Mira tío, te tomas una ampolla de Revital y adiós a tu crisis energética. Con Revital trabajas, entras, sales, invitas a tu chica, cumples, tio [...].

[Voz masculina]: ¿Qué por qué tomo Revital? Porque te pone en forma. Yo no sé si es el ging seng que lleva, la jalea o la vitamina $C$, pero me tomo una ampolla de Revital y funciono para todo el día. Y vamos, si lo tomo por la noche, aguanto lo que me echen.

Lo mismo sucede con un producto de similares características:

[Voz masculina]: Es la ley de Murphy: llegas del trabajo derrotao y ese día, precisamente ese día, a tu mujer le apetece jugar. Nada, me tomo una ampolla de Energizil y me pongo a su nivel. Con Energizil me pongo a mil. Y a jugar toda la noche $e^{31}$.

La figura femenina no está suficientemente presente en la publicidad emitida en el horario de deportes y además las masculinas adquieren otro tipo de consideración respecto a otras franjas horarias.

Las mujeres sorprenden como protagonistas de la publicidad en espacios eminentemente masculinos, como son los deportivos, en los que apenas escuchamos voces femeninas y en los que el lenguaje utilizado dificulta también el que podamos sentirnos aludidas. Todo el discurso que se utiliza en este tipo de programación deportiva parece estar dirigido a un público masculino, ciertamente oyente mayoritario de los mismos. A pesar de que con los años, las mujeres, sobre todo las jóvenes, se han empezado a interesar por estas emisiones y, a pesar de la incorporación de las periodistas

\footnotetext{
${ }^{31}$ En "Al primer toque", 21 de agosto de 2009. Emisión simultánea en Radio Marca y Onda Cero. Horario: de 24:00 a 2:00 h.
} 
deportivas a estos programas, todavía el discurso está claramente estereotipado (Alfaro, Bengoechea y Vázquez 2010: 63).

En la publicidad radiofónica estos cambios no son demasiado visibles. En la actualidad, mujeres y hombres se preocupan por el funcionamiento del coche, por optimizar los ahorros y organizar las mejores vacaciones siempre que sea posible. Pero todavía la publicidad de coches para fines profesionales sigue haciendo usos tan sexistas como utilizar estribillos como "me gustan las mulatas" o sugerir el regalo de "una nórdica" con la adquisición ${ }^{32}$. En el siguiente anuncio, la mulata se convierte en la "depredadora de un indiferente hombre blanco". Es el llamado estereotipo de Jezabel, la mujer étnica hipersexy y promiscua (Izquierdo 2008: 81):

[Voz masculina 1, cantando]: Me gustan las mulatas, me gustan las mulatas, me gustan...

[Voz masculina 2]: ¿Quieres la nueva melodía de Prosickito en tu móvil? Envía Renault espacio prosi al [...].

[Voz masculina 3]: Creemos en tu negocio tanto como tú. Por eso este mes Renault Kangoo desde 8150 euros. Más información en entreprofesionales.renault.es ${ }^{33}$.

Jesús Castañón (2009) reconoce ocho grandes retos que se presentan en el siglo XXI para la evolución del deporte y que, con el tiempo, deberán hacerse visibles también en el idioma: el segundo de ellos es la plena incorporación de las mujeres a todos los

\footnotetext{
${ }^{32}$ Es una pena que no dispongamos del anuncio completo, ya que en sí carecía de interés, excepto la frase final. Se trata de una dramatización de los presentadores del programa "Tiempo de juego": uno de ellos va a un concesionario, pero no sabe ni qué características debe tener el coche (motor, potencia...). Ahora bien, le pregunta al final al otro, que realiza el papel de vendedor: ¿me podría llevar una nórdica? En "Tiempo de juego", Cadena COPE, 14/15 de febrero de 2004. Horario: de 16:00 a 23: 30 h. Así, si la nórdica es, en el imaginario masculino mediterráneo, la mujer liberada sexualmente por excelencia y no hay más que ver alguna de las muchas películas que así lo ponen de manifiesto en nuestro cine de los años 60 y 70 o la siguiente referencia bibliográfica: Lust, Erika (2011), Por qué las suecas son un mito erótico. Un manual para conocer las armas secretas de las diosas del norte, Barcelona, Océano.

${ }^{33}$ En "El tirachinas", Cadena COPE, 4 de febrero de 2007. Horario: de 23: 25 a 1:00 h.
} 
estamentos de este ámbito, dentro y fuera de los recintos de competición. Como hemos visto en este apartado, estamos ante un aspecto aún pendiente y en el que todavía no se ven elementos claros de una evolución.

\section{EI lenguaje en la publicidad de los espacios deportivos}

Después de años analizando algunos de los principales rasgos de la publicidad que se emite en la radio española, una de las conclusiones fundamentales que se pueden adelantar es que el lenguaje utilizado en estos anuncios grabados no presenta, en la mayoría de ocasiones, unos sesgos sexistas difícilmente solucionables. Incluso se puede decir esto mismo cuando se trata de la programación deportiva, espacio poco propicio para una comprensión de las desigualdades de género que se pueden provocar con la mala utilización del lenguaje y de sus estrategias lingüísticas. Por ejemplo, en el año 2004 sorprendía encontrar el anuncio de una oferta de trabajo en la que se decía: Empresa de comunicación de Málaga busca persona con experiencia como secretaria y conocimiento en utilización de ordenadores [...], para después convocar a los interesados a enviar su currículum vitae $^{34}$. Se trata de un ejemplo de cómo las profesiones tradicionalmente vinculadas a mujeres han mantenido su terminología en femenino, creándose un dual aparente que perjudica a la consideración profesional cuando se trata del femenino (secretaria: 'mujer que se dedica a tareas subalternas'), frente al prestigio que se confiere al masculino (secretario: 'hombre que desempeña un cargo de responsabilidad') (Guerrero y Medina 2006).

En la siguiente recopilación, de 2006-2007, el lenguaje publicitario en la programación deportiva presentaba dos características muy marcadas, como eran el uso

\footnotetext{
${ }^{34}$ En la promoción de un curso de informática, dirigido a personas que puedan tener alguna relación con el trabajo con ordenadores, vemos que se utilizan masculinos genéricos que pueden hacer referencia a ambos sexos, excepto en el caso de las secretarias: Si la informática es un problema para encontrar trabajo, no se preocupe. Aprenda paso a paso todo: Word, Excel, Access, Power Point, Outlook. Lo imprescindible hoy para acceder a un puesto de trabajo o mejorar en el que ya tiene [...] Secretarias, autónomos, profesionales liberales, pequeños comerciantes, directivos, universitarios, opositores encontrarán [...] un medio de ayuda y aprendizaje [...]. En Gil Benítez (2007: 94).
} 
de los masculinos genéricos y el de vocativos como hombre o tío, sobre todo en el caso de la publicidad realizada en directo. Es una tendencia que considero relacionada con la presencia mayoritaria de voces masculinas: si los anuncios están protagonizados por hombres y están dirigidos fundamentalmente a ellos, no parece un contrasentido que se expresen en estos términos, aunque en algún momento pueden llegar a incurrir en el salto semántico, relacionado con el androcentrismo tan presente en la programación deportiva $^{35}$ :

[Voz masculina, Pepe Domingo Castaño]: ¿Cómo cantaría Funciona de Pascual un tío que toma Funciona?

[Voces masculinas, Pepe Domingo Castaño y Equipo]: [rápido] Funciona de Pascual, Funciona de Pascual.

[Voz masculina, Pepe Domingo Castaño]: Y un tío que no toma Funciona, ¿cómo lo cantaría? [...].

[Voz masculina 1, Pepe Domingo Castaño]: [...] una tarde de fútbol sin pipas? Pues dile a Facundo que pida las pipas. Pídele las pipas a Facundo.

[Voz masculina 2, responde uno del público]: No te vayas de este mundo sin probar pipas Facundo.

[Voz masculina 1, Pepe Domingo Castaño]: Muy bien dicho, sí señor. Facundo, saca las pipas, hombre. Que la gente está pidiendo pipas aquí. Tú sabes por qué las pipas, ¿cómo te llamas tú?

[Voz masculina 2, responde]: David [...].

35 Los dos anuncios en "Carrusel deportivo", Cadena Ser, 17 de diciembre de 2006. Horario: de 15: 30 a $23: 00 \mathrm{~h}$ 
En la recopilación de 2009 otras características del lenguaje publicitario radiofónico merecen la atención. Así, el primer rasgo es el uso casi generalizado del tuteo como forma de dirigirse a su público objetivo, cuando se trata de cuñas grabadas y también en la publicidad que se realiza en directo:

[Voz masculina, el presentador del programa]: [...] Es una cuestión que debes plantearte en serio: necesitas pagar menos cada mes y mejorar tu calidad de vida. Mañana jueves es un día perfecto para llamar a Freedom, estés en tu ciudad, en tu pueblo, de vacaciones o currando, porque no hay que desplazarse para pedir que estudien tu caso [...] Si ellos encuentran la forma de que ahorres, te lo dirán en pocas horas. Recuerda el número $[\ldots]^{36}$.

Parece clara la intención de interpelar directamente a oyentes y captar su atención hacia el producto que se intenta vender. Algunas veces, la manera de hacerlo es a través de los diferentes usos del pronombre tú, cuya efectividad en el ámbito publicitario ha sido estudiada por Hernández Toribio (2008).

\section{Conclusión}

La voz en la publicidad que se emite en la programación deportiva es mayoritariamente masculina. En el año 2009, el porcentaje de voces masculinas en los anuncios emitidos era del $76,1 \%$ frente al $5,1 \%$ en el caso de la femenina. En la recopilación realizada a finales de 2006 y principios 2007, la tendencia era muy similar ya que el $84,3 \%$ de los anuncios emitidos en estos espacios estaban protagonizados por voces masculinas y el $0 \%$ por voces femeninas. En 2004, las cifras fueron también

36 En "Marcador", Radio Marca, 19 de agosto de 2009. Horario: de 20:00 a 22:00 h. 
esclarecedoras: la voz que se escucha en el $72,5 \%$ de los anuncios emitidos era exclusivamente masculina, mientras que solo hay un 5,625\% en los que era femenina.

Por tanto, la situación parece que ha empeorado desde el año 2004, con unos porcentajes que ponen de manifiesto la escasa consideración de la presencia femenina en la publicidad de un tipo de programación como esta, que se centra en la retransmisión y los comentarios de fútbol, y que parece eminentemente diseñada con un carácter masculino.

Puede que haya dos motivos que ayuden a explicar esta mínima presencia de mujeres en la publicidad de la radio deportiva española: en primer lugar, la publicidad parece resistirse a una idea que resulta evidente: a las mujeres también les gusta el deporte y quieren mantenerse informadas con este tipo de programación; en segundo lugar, apenas hay voces femeninas en esta publicidad porque tampoco las hay en los equipos que realizan los programas deportivos.

Por lo que respecta a los productos publicitados, en la recopilación de 2009/10, lo más repetido será todo aquello relacionado con los coches. Le sigue en importancia la autopromoción que realizan las emisoras de sus contenidos. En tercer lugar, encontramos la restauración y demás locales relacionados con el ocio. Y ya, a mayor distancia, aparece un anunciante, las agencias de viaje, cuya posición está muy condicionada por las fechas en las que ha tenido lugar la toma de datos. En los primeros puestos tenemos también las tiendas y los centros comerciales. Seguros, empresas financieras y bancos forman igualmente una terna muy repetida en la publicidad de la programación deportiva. Si dejamos a un lado la publicidad de autopromoción radiofónica, los conceptos más repetidos están relacionados con aspectos que tradicionalmente se han reservado a la actuación de la figura masculina: el coche y su mantenimiento, el ocio, las adquisiciones que implican un mayor desembolso de dinero para el hogar y decisiones económicas de importancia.

En la recopilación realizada entre finales de 2006 y principios de 2007, aunque la variedad de productos es interesante y tampoco se puede afirmar rotundamente que unos productos se orientan en exclusividad a los hombres y otros a las mujeres, lo cierto es 
que más que en ninguna otra franja horaria, se incide en la compra de vehículos comerciales, utilitarios de gama alta, en los talleres para coches y en productos tan especializados como el cemento o, incluso, los tractores.

En el análisis de la publicidad recopilada en 2004 se llega prácticamente a la misma conclusión: la publicidad en la franja horaria deportiva es eminentemente masculina; está protagonizada por hombres, se dirige a ellos y les ofrece aquellos productos que más les interesan.

El deporte se convierte además en el espacio ideal para que el hombre se fije en su apariencia y en su bienestar físico: mientras que en franjas horarias como la matinal o la de tarde la preocupación se centra en "arreglar" los problemas que acucian a las mujeres (con su peso, con su falta de energía o con la llegada de la menopausia), en la deportiva estos consejos desaparecen y se convierte en el espacio en el que los hombres pueden buscar la solución a problemas como la pérdida del cabello, los ronquidos o dificultades sexuales. En algunos casos, además, encontramos una circunstancia muy significativa: son ellos los afectados, aunque las mujeres tienen que soportarlo de la misma manera y, en algunos casos, incluso tomar la iniciativa para su solución.

Después de años analizando algunos de los principales rasgos de la publicidad que se emite en la radio española, una de las conclusiones fundamentales que se pueden adelantar es que el lenguaje utilizado en estos anuncios grabados no presenta, en la mayoría de ocasiones, unos sesgos sexistas difícilmente solucionables. Incluso se puede decir esto mismo cuando se trata de la programación deportiva, espacio poco propicio para una comprensión de las desigualdades de género que se pueden provocar con la mala utilización del lenguaje y de sus estrategias lingüísticas.

Sin embargo, el lenguaje publicitario en la programación deportiva puede presentar dos características muy marcadas, como son el uso de los masculinos genéricos y el de vocativos como hombre o tío, sobre todo en el caso de la publicidad realizada en directo. Se trata de una tendencia que vinculo con la presencia mayoritaria de voces masculinas: si los anuncios están protagonizados por hombres y están dirigidos fundamentalmente a ellos, no parece un contrasentido que se expresen en estos términos, aunque en algún 
momento pueden llegar a incurrir en el salto semántico, relacionado con el androcentrismo tan presente en este tipo de programación.

\section{Referencias bibliográficas}

Alfaro Gandarillas, Elida; Mercedes Bengoechea Bartolomé y Benilde Vázquez Gómez. 2010. Hablamos de deporte. En femenino y en masculino, Serie Lenguaje $n^{0} 7$. http://www.csd.gob.es/csd/estaticos/myd/hablamos_deporte.pdf [22 de enero de 2013].

Álvarez Villa, Ángels. 2004. "La creatividad publicitaria en los magacines deportivos radiofónicos locales". La publicidad local. III Congreso de Comunicación Local (ComLoc 2003). Eds. Rafael López Lita, Francisco Fernández Beltrán y Ángeles Durán Mañes. Castelló de la Plana: Universitat Jaume I. 173-185.

Arenas Llopis, Luis et al. 2003. ARESTE: Arrinconando estereotipos en los medios de comunicación y la publicidad. Madrid: Dirección General de la Mujer.

Castañón Rodríguez, Jesús. 2009. "Recursos lingüísticos en la radio deportiva". Idioma $y$ Deporte, 118. http://www.idiomaydeporte.com/radiorec.htm [24 de mayo de 2013].

Gil Benítez, Eva Mª 2007a. "Los estereotipos de la mujer en la publicidad radiofónica". Mujeres, simbolismo y vida. $\mathrm{M}^{\mathrm{a}}$ de los Ángeles Durán. Málaga: Universidad de Málaga. 79-96.

. 2007b."El sexismo en la publicidad radiofónica. Lo dicho y lo sobreentendido". Avanzando hacia la igualdad en las Ciencias Sociales. Coord. Susana Guerrero Salazar. Málaga: Diputación de Málaga/Asociación de Estudios Históricos sobre la Mujer. 45-57.

. 2008. "El lenguaje en los mensajes publicitarios: Sexismo lingüístico/Sexismo social". $2^{\circ}$ Congreso Internacional de Estudios de Género y políticas de Igualdad: "La imagen pública de la mujer", Sevilla: Instituto Andaluz de la Mujer. 1-15. http://www.iam-congresopoliticasdegenero.es/descarga/jueves_27(9_10).pdf [22 de febrero de 2011].

. 2010. "La publicidad electoral con una perspectiva de género. Análisis de tres campañas electorales $(2004,2007,2009)$ ". Estudios actuales sobre lengua, literatura y su didáctica. Homenaje a Emilio A. Núñez Cabezas. Eds. Raúl Cremades García, Cristóbal González Álvarez y Susana Guerrero Salazar. Málaga: VG Ediciones/Universidad. 223-242. 
. 2012a. "La presencia de las mujeres en la publicidad radiofónica de la programación deportiva (2004-2010)". El discurso deportivo en los medios de comunicación. Coords. Susana Guerrero Salazar y Raúl Cremades García. Málaga: VG Ediciones. 133-148.

. 2012b. "La utilización de la imagen femenina en la auto-promoción de una emisora de radio: el 95.2 FM del dial de Málaga (Radio Chanquete”. Simposio Mujeres y Ciencia. Historia, retos y perspectivas. Coord. Carmen Cortés Zaborras. Málaga: Perséfone. 55-59. http://www.aehm.uma.es/ACTAS_MYC_ISBN.pdf [17 de diciembre de 2012].

Gómez Sánchez, Ma Elena. 2012. “...Y además, deportistas. Algunos ejemplos del discurso mediático sobre las mujeres en el periodismo deportivo". El discurso deportivo en los medios de comunicación. Coords. Susana Guerrero Salazar y Raúl Cremades García. Málaga: VG Ediciones. 115-132.

Guerrero Salazar, Susana y Antonia $\mathrm{M}^{\mathrm{a}}$ Medina Guerra. 2006. Guía para un uso igualitario del lenguaje en las corporaciones locales. "Hablemos en igualdad". Almería: Servicio Provincial de Mujeres.

Hernández Toribio, $\mathrm{M}^{\mathrm{a}}$ Isabel. 2008. "La lengua de la publicidad: algunas estrategias al servicio de la persuasión emocional". La lengua española en los medios de comunicación y en las nuevas tecnologías. Ed. Antonio Arroyo Almaraz. Barcelona: Laberinto. 221-252.

Herrero Gutiérrez, Francisco Javier y David Rodríguez Ramos. 2009. "La locución de los narradores deportivos radiofónicos en España", Revista Latina de Comunicación Social, 64. 968-987. http://www.revistalatinacs.org/09/art/874_Salamanca/75_101_Herrero.htm [24 de mayo de 2013].

Herrero Gutiérrez, Francisco Javier e Hilario José Romero Bejarano. 2011. "Formatos publicitarios en la radio deportiva: de la tradicional cuña publicitaria a la importancia de la figura del animador". Pensar la Publicidad, vol. 5, n 2. 233-254. http://dx.doi.org/10.5209/rev_PEPU.2011.v5.n2.37871 [24 de mayo de 2013].

Izquierdo Iranzo, Patricia. 2008. "Representación de la minoría entre la minoría: relaciones inter-género e inter-étnicas en la publicidad". La representación/presencia de la mujer en los Medios de Comunicación, Feminismo/s, Revista del Centro de Estudios sobre la Mujer, 11. Coord. Sonia Núñez Puente y ed. Helena Establier Pérez. Alicante: Universidad. 73-94.

Pérez Chico, Ma Ángeles. 2002. "Modelos publicitarios". Mujeres, hombres y medios de comunicación. Valladolid: Dirección General de la Mujer e Igualdad de Oportunidades. 43-53 (Tomo I: Ponencias).

Rojas Torrijos, José Luis. 2010. "La construcción de las noticias deportivas desde una mirada androcéntrica. De la invisibilidad a los estereotipos de la mujer deportista". 
Vivat

Academia,

113.

http://www.ucm.es/info/vivataca/numeros/n113/DATOSS.htm [20 de mayo de 2013].

Vigara Tauste, Ana Ma . 2008. "Periodismo deportivo en España: sexismo discursivo y discriminación de género". Nuevas tendencias de la lengua española en los medios de comunicación. Coords. Susana Guerrero Salazar y Emilio Alejandro Núñez Cabezas. Málaga: VG Ediciones. 113-160. 